\title{
A NOTE ON THE DERIVATIVES OF INTEGRAL FUNCTIONS
}

\author{
S. M. SHAH
}

1. Introduction. Let $f(z)=\sum_{0}^{\infty} a_{n} z^{n}$ be an integral function of order $\rho$ and lower order $\lambda$, and $M(r)=\max _{|z|=r}|f(z)| ; M^{\prime}(r)=\max _{|z|=r}\left|f^{\prime}(z)\right|$. In a recent paper [1] $]^{1}$ I have proved the following two theorems.

Theorem A. If $f(z)$ be any integral function of order $\rho$ then ${ }^{2}$

$$
\limsup _{r \rightarrow \infty} \frac{\log \left\{r M^{\prime}(r) / M(r)\right\}}{\log r}=\rho .
$$

THEOREM B. If $f(z)=\sum a_{n} z^{n}$ be an integral function of lower order $\lambda$ and $a_{n} \geqq 0$ then

$$
\liminf _{r \rightarrow \infty} \frac{\log \left\{r M^{\prime}(r) / M(r)\right\}}{\log r}=\lambda .
$$

The condition that the coefficients $a_{n}$ be real and non-negative is unnecessary. The purpose of this note is to prove the following two theorems and to deduce a number of interesting results.

THEOREM 1. If $f(z)$ be an integral function of lower order $\lambda(0 \leqq \lambda \leqq \infty)$ then

$$
\liminf _{r \rightarrow \infty} \frac{\log \left\{r M^{\prime}(r) / M(r)\right\}}{\log r}=\lambda .
$$

TheOREM 2. For any integral function $f(z)$ we have

$$
\begin{aligned}
\liminf _{r \rightarrow \infty} M^{\prime}(r) / M(r) & \leqq \liminf _{r \rightarrow \infty} \nu(r) / r \leqq \limsup _{r \rightarrow \infty} \nu(r) / r \\
& \leqq \limsup _{r \rightarrow \infty} M^{\prime}(r) / M(r),
\end{aligned}
$$

$$
\begin{array}{cc}
\liminf _{r \rightarrow \infty} M^{(s+1)}(r) / M^{(s)}(r) \leqq \liminf _{r \rightarrow \infty} \nu(r) / r \leqq & \limsup _{r \rightarrow \infty} \nu(r) / r \\
\leqq & (s=1,2,3, \cdots),
\end{array}
$$

where $f^{(s)}(z)$ is the sth derivative of $f(z), M^{(s)}(r)=\max _{|z|=r}\left|f^{(s)}(z)\right|$

Received by the editors January 6, 1947, and, in revised form, April 1, 1947.

${ }^{1}$ Numbers in brackets refer to the bibliography at the end of the paper.

${ }^{2}$ A glance at the proof $[1$, pp. 1-2] shows that the result (1.1) holds when $\rho$ is infinite. An alternative proof of Theorem $A$ is to employ Lemma 4 and relation (8) of my paper [1, p. 1]. 
and $\nu(r)$ is the rank of the maximum term of $f(z)$ for $|z|=r$.

\section{Lemmas.}

\section{LEMMA 1. Let}

(2.1) $F(x)$ be a positive nondecreasing function for $x>x_{0}$,

$$
\liminf _{x \rightarrow \infty} F(x) / x=a \quad(0 \leqq a<\infty) ;
$$

then, corresponding to each pair of positive numbers $b, c$ satisfying the inequalities

$$
a<b ; \quad a / b<c<1
$$

there is a sequence $x_{1}, x_{2}, \cdots$, tending to infinity such that

$$
F(x)<b x \quad\left(c x_{n} \leqq x \leqq x_{n}\right) .^{3}
$$

PROOF. For there is a sequence $x_{1}, x_{2} \cdots$ such that

$$
F\left(x_{n}\right)<b c x_{n}
$$

Hence if $c x_{n} \leqq x \leqq x_{n}$

$$
F(x) \leqq F\left(x_{n}\right)<b c x_{n} \leqq b x .
$$

LEMMA 2. Let (2.1) hold,

$$
\limsup _{x \rightarrow \infty} F(x) / x=a_{1} \quad\left(0<a_{1} \leqq \infty\right) ;
$$

then, corresponding to each pair of positive numbers $b_{1}, c_{1}$, satisfying the inequalities

$$
b_{1}<a_{1} ; \quad 1<c_{1}<a_{1} / b_{1}
$$

there is a sequence $X_{1}, X_{2}, \cdots$ tending to infinity such that

$$
F(x)>b_{1} x \quad\left(X_{n} \leqq x \leqq c_{1} X_{n}\right) .
$$

For there is a sequence $\left\{X_{n}\right\}$ tending to infinity such that

$$
F\left(X_{n}\right)>b_{1} c_{1} X_{n} \text {. }
$$

If $X_{n} \leqq x \leqq c_{1} X_{n}$

$$
F(x) \geqq F\left(X_{n}\right)>b_{1} c_{1} X_{n} \geqq b_{1} x \text {. }
$$

LEMma 3 [2, p. 22]. Let (2.1) hold,

\footnotetext{
${ }^{3}$ It is always possible to choose the sequence $\left\{x_{n}\right\}$ such that two consecutive intervals have no common point. Similarly for the intervals of Lemmas 2, 3, and 4 .
} 


$$
\liminf _{x \rightarrow \infty} \log F(x) / \log x=\alpha
$$$$
(0 \leqq \alpha<\infty)
$$

then, corresponding to each pair of positive numbers $\beta, \gamma$ satisfying the inequalities

$$
\alpha<\beta ; \quad \alpha / \beta<\gamma<1
$$

there is a sequence $y_{1}, y_{2}, \cdots$ tending to infinity such that

$$
F(x)<x^{\beta} \quad\left(y_{n}^{\gamma} \leqq x \leqq y_{n}\right) .
$$

LEMMA 4. Let (2.1) hold,

$$
\limsup _{x \rightarrow \infty} \log F(x) / \log x=\alpha_{1} \quad\left(0<\alpha_{1} \leqq \infty\right) ;
$$

then, corresponding to each pair of positive numbers $\beta_{1}, \gamma_{1}$, satisfying the inequalities

$$
\beta_{1}<\alpha_{1} ; \quad 1<\gamma_{1}<\alpha_{1} / \beta_{1}
$$

there is a sequence $Y_{1}, Y_{2}, \cdots$ tending to infinity such that ${ }^{4}$

$$
F(x)>x^{\beta_{1}} \quad\left(Y_{n} \leqq x \leqq Y_{n}^{\gamma_{1}}\right) .
$$

For there is a sequence $\left\{Y_{n}\right\}$ such that

$$
\log F\left(Y_{n}\right)>\beta_{1} \gamma_{1} \log Y_{n}
$$

If $Y_{n} \leqq x \leqq Y_{n}^{\gamma_{1}}$

$\log F(x) \geqq \log F\left(Y_{n}\right)>\beta_{1} \gamma_{1} \log Y_{n}=\beta_{1} \log Y_{n}^{\gamma_{1}} \geqq \beta_{1} \log x$.

3. Proof of Theorem 1. Let

$$
\theta(r)=\frac{\log \left\{r M^{\prime}(r) / M(r)\right\}}{\log r} .
$$

If $\lambda$ be infinite, we have from the inequality [3]

$$
M^{\prime}(r)>(M(r) \log M(r)) /(r \log r) ; \quad r>r_{0}=r_{0}(f)
$$

that ${ }^{5}$

$$
\liminf _{r \rightarrow \infty} \theta(r)=\infty \text {. }
$$

We therefore suppose that $0 \leqq \lambda<\infty$. From the inequality (3.1) it follows that

$$
\lim _{r \rightarrow \infty} \inf \theta(r) \geqq \lambda .
$$

4 We can deduce Lemmas 1 and 2 from Lemmas 3 and 4.

$5 r_{0}$ and $n_{0}$ are not necessarily the same at each occurrence. 
If $\nu(r)$ denotes the rank of the maximum term of $f(z)$, then we have [2, p. 21]

$$
\lim _{r \rightarrow \infty} \inf \log \nu(r) / \log r=\lambda \text {. }
$$

Hence by Lemma 3, corresponding to each pair of positive numbers $\beta, \gamma$ satisfying the inequalities $\lambda<\beta ; \lambda / \beta<\gamma<1$, there is a sequence $y_{1}, y_{2}, \cdots$ tending to infinity such that

$$
\nu(r)<r^{\beta} \quad\left(y_{n}^{\gamma} \leqq r \leqq y_{n}\right) .
$$

Let $E_{n}$ denote the set of points $r\left(y_{n}^{r} \leqq r \leqq y_{n}\right)$ and $E=E_{1}+E_{2}+\cdots$. Let $F$ denote the set of points $r$ which lie [4, p. 105] outside a set of exceptional segments in which, for $r>R$, the variation of $\log r$ is less than $K \nu(R / k)^{-1 / 12}$. Since the variation of $\log r$ in $E_{n}$ is

$$
\log y_{n}-\gamma \log y_{n}=(1-\gamma) \log y_{n},
$$

which tends to infinity with $n$, there are points in $E_{n}$ which do not belong to the set of exceptional segments. The set $E F$ therefore contains a sequence $e_{1}, e_{2}, \cdots$ tending to infinity. At these points [4, p. 105] $e_{n}$

$$
\begin{aligned}
r M^{\prime}(r) & \sim M(r) \nu(r), & r=e_{n} \\
r M^{(s+1)}(r) & \sim M^{(s)}(r) \nu(r) . &
\end{aligned}
$$

Hence $^{5}$ for $n>n_{0}$

$$
e_{n} M^{\prime}\left(e_{n}\right) / M\left(e_{n}\right)<2 \nu\left(e_{n}\right)<2 e_{n}^{\beta}
$$

and so $\lim \inf _{r \rightarrow \infty} \theta(r) \leqq \beta$ and since $\beta-\lambda$ can be chosen arbitrarily small we have

$$
\liminf _{r \rightarrow \infty} \theta(r) \leqq \lambda
$$

and so $\lim \inf _{r \rightarrow \infty} \theta(r)=\lambda$.

4. Proof of Theorem 2. Let $\lim \inf _{r \rightarrow \infty} \nu(r) / r=a$ and suppose first that $a<\infty$. Then if

$$
a<b, \quad a / b<c<1,
$$

we have, by Lemma $1, \nu(r)<b r\left(c x_{n} \leqq r \leqq x_{n}\right)$. Let $E_{n}$ denote the set of points $r\left(c x_{n} \leqq r \leqq x_{n}\right)$ and $E=E_{1}+E_{2}+\cdots$. The variation of $\log r$ in $E_{n}$ is $\log x_{n}-\log c x_{n}=\log 1 / c$ which is not less than $K \nu(R / k)^{-1 / 12}$ if $R$ be large enough. The total variation of $\log r$ in the intervals $\sum_{p=1}^{n} E_{p}$ tends to infinity with $n$. Hence the set $E F$ contains [4, p. 105] 
a sequence $e_{1}^{\prime}, e_{2}^{\prime}, \cdots$ tending to infinity. For $r=e_{n}^{\prime}\left(n>n_{0}\right)$

$$
\begin{aligned}
M^{\prime}(r) / M(r) & \sim \nu(r) / r<b, \\
M^{(s+1)}(r) / M^{(s)}(r) & \sim \nu(r) / r<b \quad(s=1,2,3, \cdots) .
\end{aligned}
$$

Hence

$$
\lim _{r \rightarrow \infty} M^{\prime}(r) / M(r) \leqq b
$$

Since $b-a$ can be chosen arbitrarily small

$$
\underset{r \rightarrow \infty}{\liminf } M^{\prime}(r) / M(r) \leqq a
$$

which certainly holds if $a=\infty$. Also

$$
\liminf _{r \rightarrow \infty} M^{(s+1)}(r) / M^{(s)}(r) \leqq a \quad(s=1,2, \cdots) .
$$

Let $\lim \sup _{r \rightarrow \infty} \nu(r) / r=a_{1}$ and suppose $a_{1}>0$. Let $b_{1}<a_{1}, 1<c_{1}<a_{1} / b_{1}$. If $G$ denotes the set of points formed by the intervals of Lemma 2 the set $G F$ contains a sequence $g_{1}, g_{2}, \cdots, g_{n}, \cdots$ tending to infinity. For $r=g_{n}\left(n>n_{0}\right)$

$$
\begin{aligned}
M^{\prime}(r) / M(r) & \sim \nu(r) / r>b_{1}, \\
M^{(s+1)}(r) / M^{(s)}(r) & \sim \nu(r) / r>b_{1} \quad(s=1,2, \cdots) .
\end{aligned}
$$

Hence $\lim \sup _{r \rightarrow \infty} M^{\prime}(r) / M(r) \geqq a_{1}$,

$$
\limsup _{r \rightarrow \infty} M^{(s+1)}(r) / M^{(s)}(r) \geqq a_{1}
$$

which hold if $a_{1}=0$. Hence the theorem follows.

5. Applications. We have from (1.1) and (1.2)

$$
\begin{aligned}
\limsup _{r \rightarrow \infty} \frac{\log \nu(r)}{\log r}=\rho & =1+\limsup _{r \rightarrow \infty} \frac{\log \left\{M^{\prime}(r) / M(r)\right\}}{\log r} \\
& =1+\limsup _{r \rightarrow \infty} \frac{\log \left\{M^{(s+1)}(r) / M^{(s)}(r)\right\}}{\log r}
\end{aligned}
$$

$$
\begin{aligned}
\liminf _{r \rightarrow \infty} \frac{\log \nu(r)}{\log r}=\lambda & =1+\liminf _{r \rightarrow \infty} \frac{\log \left\{M^{\prime}(r) / M(r)\right\}}{\log r} \\
& =1+\liminf _{r \rightarrow \infty} \frac{\log \left\{M^{(s+1)}(r) / M^{(s)}(r)\right\}}{\log r} \\
& (s=1,2, \cdots) .
\end{aligned}
$$


Let $s$ denote any fixed positive integer and $C, C_{1}$ two positive constants.

(5.3) If $M^{\prime}(r) \geqq C M(r)$ for all $r>r_{0}$ then either $\lambda>1$ or $\lambda=1$ and $\lim \inf _{r \rightarrow \infty} \nu(r) / r \geqq C$.

From (1.3) we have $\lim \inf _{r \rightarrow \infty} \nu(r) / r \geqq C$. Hence $\lambda \geqq 1$.

REMARK (i). This is a best possible result in the sense that there are functions for which $M^{\prime}(r) \geqq C M(r)$ and $\nu(r) \sim C r$. We may take for instance $f(z)=\exp (C z)$.

(ii) The converse-if $\lim \inf _{r \rightarrow \infty} \nu(r) / r \geqq C$ then $M^{\prime}(r) \geqq C M(r)$ for all $r>r_{0}$-is false. For consider $f(z)=\cosh C z$. Here $\lambda=1=\rho, \nu(r) \sim C r$.

$$
\begin{array}{lll}
\frac{M^{\prime}(r)}{M(r)}=C \frac{e^{C r}-e^{-C r}}{e^{C r}+e^{-C r}}<C & \text { for all } r>0, \\
\frac{M^{\prime \prime}(r)}{M^{\prime}(r)}=C \frac{e^{C r}+e^{-C r}}{e^{C r}-e^{-C r}}>C & \text { for all } r>0,
\end{array}
$$

and so on.

(5.4) If $M^{(s+1)}(r) \geqq C M^{(s)}(r), r>r_{0}$, then $\lambda \geqq 1$ and $\lim \inf _{r \rightarrow \infty} \nu(r) / r$ $\geqq C$.

(5.5) If $M^{\prime}(r) \leqq C_{1} M(r)$, or if $M^{(s+1)}(r) \leqq C_{1} M^{(s)}(r)$, for all $r>r_{0}$, then either $\rho<1$ or $\rho=1$ and $\lim \sup _{r \rightarrow \infty} \nu(r) / r \leqq C_{1}$. This follows from Theorem 2. If $f(z)$ is of order 1 then $[5, \mathrm{p} .81]$ it follows that $\lim \sup _{r \rightarrow \infty} \log M(r) / r \leqq C_{1}$.

Let $\phi(r)$ be any function, nondecreasing and positive for $r>r_{0}$, and such that $\log \phi(r) / \log r$ tends to zero as $r$ tends to infinity.

(5.6) If $M^{\prime}(r) \geqq(1 / \phi(r)) M(r)$, or if $M^{(s+1)}(r) \geqq(1 / \phi(r)) M^{(s)}(r)$ for a sequence of values of $r$ tending to infinity, then $\rho \geqq 1$.

This follows from (5.1). If this hypothesis holds for all $r>r_{0}$ then from (5.2) we get $\lambda \geqq 1$.

(5.7) If $M^{\prime}(r) \leqq \phi(r) M(r)$, or if $M^{(s+1)}(r) \leqq \phi(r) M^{(s)}(r)$, for $a$ sequence of values of $r$ tending to infinity, then $\lambda \leqq 1$.

This follows from (5.2). If this hypothesis holds for all $r>r_{0}$ then from (5.1) we get $\rho \leqq 1$.

(5.8) If $1 / \phi(r) \leqq M^{\prime}(r) / M(r) \leqq \phi(r)$ or if $1 / \phi(r) \leqq M^{(s+1)}(r) / M^{(s)}(r)$ $\leqq \phi(r)$ for all $r>r_{0}$ then $\lambda=\rho=1$.

This follows from (5.6) and (5.7).

(5.9) If $\rho<1$ then

$$
M(r)>\phi(r) M^{\prime}(r)>\phi^{2}(r) M^{\prime \prime}(r)>\cdots>\{\phi(r)\}^{8} M^{(s)}(r)
$$

for all $r>r_{0}$.

This follows ${ }^{6}$ from (5.1).

C Cf. (5.6) above. 
(5.10) If $\lambda>1$ then

$$
M(r)<\frac{1}{\phi(r)} M^{\prime}(r)<\frac{1}{\phi^{2}(r)} M^{\prime \prime}(r)<\cdots<\frac{1}{\{\phi(r)\}^{s}} M^{(s)}(r)
$$

for all $r>r_{0}$

This follows ${ }^{7}$ from (5.2).

(5.11) If $\lambda=1$ and $\lim \inf _{r \rightarrow \infty} \nu(r) /(r \log r)>1$ then $^{8} M(r)<M^{\prime}(r)$ $<M^{\prime \prime}(r)<\cdots<M^{(s)}(r)$ for all $r>r_{0}$. If $\lim \inf _{r \rightarrow \infty} \nu(r) /(r \log r)$ $=l>1$ then since

$$
\begin{gathered}
\log M(r)>\int_{r_{\theta}}^{r}\{\nu(t) / t\} d t, \\
\liminf _{r \rightarrow \infty} \log M(r) /(r \log r)>1 .
\end{gathered}
$$

Hence for all $r>r_{0}$

$$
\begin{aligned}
M^{\prime}(r) / M(r) & >\log M(r) /(r \log r)>1, \\
M^{\prime \prime}(r) / M^{\prime}(r) & >\log M^{\prime}(r) /(r \log r)>\log M(r) /(r \log r)>1,
\end{aligned}
$$

and so on.

(5.12) If $\lambda=1$ and $\lim \inf _{r \rightarrow \infty} \nu(r) / r<1$ there is a sequence of numbers $r$ tending to infinity for which

$$
M(r)>M^{\prime}(r)>\cdots>M^{(s)}(r) .
$$

Let $\lim \inf _{r \rightarrow \infty} \nu(r) / r=a$ and $a<b<1$. The result follows from (4.1) and (4.2). This result does not hold if $\lim _{\inf _{r \rightarrow \infty} \nu} \nu(r) / r \geqq 1$. In fact for the function $f(z)=\cosh z, \nu(r) \sim r$ and the sequence $\left\{M^{(n)}(r)\right\}$ $(n=0,1,2, \cdots)$ is not monotonic for any $r>0$.

(5.13) If $\lambda<1$, there is a sequence of numbers $r$ tending to infinity for which

$$
M(r)>\phi(r) M^{\prime}(r)>\phi^{2}(r) M^{\prime \prime}(r)>\cdots>\{\phi(r)\}^{s} M^{(s)}(r) .
$$

This follows from (3.3) and (3.4).

(5.14) If $\rho=1$ and $\lim \sup _{r \rightarrow \infty} \nu(r) / r>1$, there is a sequence of numbers $r$ tending to infinity for which

$$
M(r)<M^{\prime}(r)<M^{\prime \prime}(r)<\cdots<M^{(s)}(r) .
$$

This follows from (4.3) and (4.4). It does not hold if $\lim \sup _{r \rightarrow \infty} \nu(r) / r \leqq 1$.

(5.15) If $\rho>1$, there is a sequence of numbers $r$ tending to infinity

" Cf. (5.7) above.

${ }^{8} \mathrm{~S}$. K. Bose has proved (5.11) with the hypothesis $\lambda>2$. 
for which

$$
M(r)<\frac{1}{\phi(r)} M^{\prime}(r)<\frac{1}{\phi^{2}(r)} M^{\prime \prime}(r)<\cdots<\frac{1}{\{\phi(r)\}^{s}} M^{(s)}(r) .
$$

Let $1<\beta_{1}<\rho ; 1<\gamma_{1}<\rho / \beta_{1} ; H$ the set of intervals of Lemma 4 . The set $F H$ contains a sequence of numbers $r$ tending to infinity for which the above inequality holds.

\section{BIBLIOGRAPHY}

1. S. M. Shah, A note on the maximum modulus of the derivative of an integral function, Journal of the University of Bombay vol. 13 (1944) pp. 1-3.

2. J. M. Whittaker, The lower order of integral functions, J. London Math. Soc. vol. 8 (1933) pp. 20-27.

3. T. Vijayaraghavan, On derivatives of integral functions, J. London Math. Soc. vol. 10 (1935) pp. 116-117.

4. G. Valiron, Lectures on the general theory of integral functions, 1923.

5. S. M. Shah, The maximum term of an entire series, Mathematics Student (Madras) vol. 10 (1942) pp. 80-82.

MUSLIM UNIVERSITY 